\title{
Onset, Maintenance, and Cessation of Effect of Galcanezumab for Prevention of Migraine: A Narrative Review of Three Randomized Placebo- Controlled Trials
}

\author{
Dulanji K. Kuruppu · James M. North • Amy J. Kovacik • \\ Yan Dong · Eric M. Pearlman · Susan L. Hutchinson
}

Received: November 2, 2020 / Accepted: January 15, 2021 / Published online: February 5, 2021

(C) The Author(s) 2021

\begin{abstract}
Introduction: Galcanezumab, a humanized monoclonal antibody that binds to calcitonin gene-related peptide, is approved for the preventive treatment of migraine in adults. It is self-administered once monthly as a subcutaneous injection. This paper describes the time course of effect of galcanezumab in patients with episodic and chronic migraine.

Methods: Data were based on three doubleblind, placebo-controlled, phase 3 studies. Patients (1773 episodic and 1113 chronic) were randomized (2:1:1) to monthly doses of placebo, galcanezumab $120 \mathrm{mg}$ with a $240 \mathrm{mg}$ loading dose, or galcanezumab $240 \mathrm{mg}$ (January 2016-March 2017). Onset of effect was determined using a sequential analysis approach based on earliest time point at which
\end{abstract}

Supplementary Information The online version contains supplementary material available at https:// doi.org/10.1007/s12325-021-01632-x.

D. K. Kuruppu ( () ) A. J. Kovacik · Y. Dong ·

E. M. Pearlman

Eli Lilly and Company, Indianapolis, IN, USA

e-mail: kuruppu_dulanji@lilly.com

J. M. North

Health Center on Broad Street, Glens Falls, NY, USA

S. L. Hutchinson

Orange County Migraine and Headache Center,

Irvine, CA, USA galcanezumab achieved and subsequently maintained statistical superiority to placebo. Maintenance of effect was a comparison of the percentages of galcanezumab- and placebotreated patients with maintenance of at least $50 \%$ response at the individual patient level. Cessation of effect was determined during a 4-month post-treatment period on the basis of change from baseline in monthly migraine headache days.

Results: Galcanezumab led to a lower percentage of patients who had a migraine headache on the first day after injection, provided maintenance of effect throughout the duration of the double-blind treatment period, and gradually lost effect without signs of rebound headache throughout the post-treatment period in most patients with episodic and chronic migraine.

Conclusion: Galcanezumab is a novel preventive therapeutic option for adult patients with migraine that has early onset of action, maintenance of effect, and gradual reduction of effect upon treatment cessation.
Trial
Registration: ClinicalTrials.gov:
NCT02614183 (EVOLVE-1); NCT02614196 (EVOLVE-2); NCT02614261 (REGAIN).

Keywords: Cessation; CGRP antagonist; Galcanezumab; Migraine; Migraine prevention; Maintenance; Onset 


\section{Key Summary Points}

Migraine is a common disabling neurologic condition that is often underrecognized, undertreated, and associated with low response rates and poor persistence on current oral standard-ofcare preventive therapies.

Development of new preventive medications for migraine has evolved on the basis of understanding the central role of calcitonin gene-related peptide (CGRP), a neuropeptide released from the trigeminal nerve during a migraine attack, in precipitating the cascade of impairing symptoms.

The exploratory analyses demonstrate the effectiveness of galcanezumab, a oncemonthly self-administered monoclonal antibody that binds to CGRP, based on onset of effect, maintenance of effect, and cessation of effect, in patients with episodic and chronic migraine.

Galcanezumab had an onset of effect beginning as early as the first day after injection for patients with episodic or chronic migraine; maintenance of effect throughout the treatment period (6 months for patients with episodic migraine and 3 months for patients with chronic migraine); and gradual reduction of effect without signs of rebound headache during the 4-month posttreatment period for the majority of patients with episodic and chronic migraine.

Galcanezumab's early onset of effect, maintenance of effect over time, and favorable tolerability profile make it a viable migraine preventive option that has the potential to improve persistence and quality of life.

\section{DIGITAL FEATURES}

This article is published with digital features, including a summary slide, to facilitate understanding of the article. To view digital features for this article go to https://doi.org/10.6084/ m9.figshare.13562183.

\section{INTRODUCTION}

Migraine, which affects about $12 \%$ of adults [1], is associated with such overwhelming and disabling symptoms [2] that it ranks as the leading cause of years lived with disability in patients aged 15-49 years and second only to low back pain in patients older than 49 years [3]. People with migraine can experience impaired function across all domains of life, and these impairments are not limited to headache days but can both precede and follow a migraine attack. Migraine is classified as episodic (up to 14 migraine headache days/month) or chronic (15 or more headache days/month for more than 3 months, of which at least 8 of those days have features of migraine headache) [2]. Such classifications are not static, and patients can move from one class to another over time.

Acute (abortive) therapy is appropriate and indicated for any number of migraine headache days/month [4], whereas preventive treatment should be offered to patients with four or more migraine headache days/month with some degree of headache-related impairment [5]. Unfortunately, only one in three patients who qualify for preventive therapy actually receive such treatment [1]. The reasons for undertreatment with preventive therapy are multifactorial. Until recently, all preventive therapies were "borrowed" from other disease states such as mood disorders, hypertension, and epilepsy and are associated with low response rates and high rates of discontinuation $[6,7]$. In a retrospective analysis of migraine preventive prescriptions in a US claims database, among patients who initiated preventive therapy, a sharp decline in persistence was observed after the first 30 days [6]. Another study evaluated cycling of preventive migraine medications from a claims database and found that in 
patients with episodic and chronic migraine, more than $75 \%$ of patients switched or discontinued their initial preventive treatment within 12 months, leading to increased healthcare resource utilization and cost [7]. Lack of consistently effective preventive medications leads to patients with migraine suffering from stigmatization and loss of hope. The ideal preventive medication would have an early onset of effect and maintenance of effect, a good tolerability/safety profile supporting persistence, and improvement of overall quality of life [8].

Recognition of the role of calcitonin generelated peptide (CGRP), a neuropeptide released from the trigeminal nerve during a migraine attack, in precipitating the cascade of impairing symptoms, has been pivotal in the development of new target-specific migraine preventive treatment options. Although the pathophysiology of migraine is complex, it is thought that stimulation of the trigeminovascular system leads to a release of neuropeptides, including CGRP, which binds to CGRP receptors and leads to a cascade of activation of nociceptors, inflammation, and vasodilatation [9].

Galcanezumab, a humanized monoclonal antibody, potently and selectively binds to the CGRP ligand and blocks its binding to the receptor $[10,11]$. Galcanezumab is approved for the preventive treatment of migraine in adults. Galcanezumab became available in the USA in September 2018 and is self-administered as a once-monthly subcutaneous injection using an auto-injector or prefilled syringe [12]. The recommended and approved dosage of galcanezumab for migraine is an initial $240 \mathrm{mg}$ loading dose (two consecutive subcutaneous injections of $120 \mathrm{mg}$ each), followed by a monthly dose of $120 \mathrm{mg}$ injected subcutaneously [12]. The use of $240 \mathrm{mg}$ as a monthly dose is not currently approved in the USA because it did not demonstrate superiority over the $120 \mathrm{mg}$ monthly dose.

The efficacy, safety, and tolerability of galcanezumab for migraine prevention were established in three phase 3 , randomized, double-blind, placebo-controlled studies in about 2800 patients with episodic migraine (EVOLVE1/EVOLVE-2) and chronic migraine (REGAIN) [13-15]. In all three pivotal studies, patients treated with monthly galcanezumab $120 \mathrm{mg}$ (with a $240 \mathrm{mg}$ loading dose) or $240 \mathrm{mg}$ doses had a statistically significantly greater overall mean reduction from baseline in the number of monthly migraine headache days vs placebotreated patients [13-15]. Overall, there were about 4.5 fewer monthly migraine headache days among galcanezumab-treated patients who participated in these studies. Extrapolated out to 1 year of treatment, the reduction in migraine headache days would equate to about 8 weeks of fewer migraine headache days/year. Over half of patients in the episodic migraine trials and over a quarter of patients in the chronic migraine trial who received galcanezumab achieved a 50\% response rate. Galcanezumab was well tolerated with low discontinuation rates, and the most common adverse event was injection site reactions [13-15].

This paper describes the time course of effect of galcanezumab in patients with episodic and chronic migraine, including onset of effect following administration of the first dose, maintenance of effect over time, and gradual cessation of effect following discontinuation of treatment.

\section{METHODS}

\section{Study Design}

Analyses were based on data from three doubleblind, randomized, phase 3 studies (January 2016-March 2017) comparing galcanezumab vs placebo in patients with episodic migraine (EVOLVE-1/EVOLVE-2) [13, 14] and chronic migraine (REGAIN) [15] that used similar study designs. For each study, patients were randomized $(2: 1: 1)$ to monthly doses of placebo, galcanezumab $120 \mathrm{mg}$, or galcanezumab $240 \mathrm{mg}$. All patients randomized to galcanezumab $120 \mathrm{mg}$ received $240 \mathrm{mg}$ as the first dose. Study treatment was administered once monthly for 3 months (REGAIN) to 6 months (EVOLVE-1/ EVOLVE-2) during the double-blind period. After treatment completion or discontinuation, patients with episodic migraine entered a 4-month post-treatment period (months 7-10), 
whereas patients with chronic migraine entered a 9-month open-label extension period (months 4-12) followed by a 4-month posttreatment period (months 13-16). Study protocols were reviewed and approved by the institutional review board for each study site. Studies were conducted according to Good Clinical Practice and to the Helsinki Declaration of 1964 and its later amendments. As this was a retrospective analysis using data that had been previously collected, participants were not required to provide formal Consent to Release Information forms. Consent and ethics reviews were performed in the original studies. A detailed list of ethical review boards can be found in the supplementary material.

\section{Patient Selection}

Study participants were adults (18-65 years) with at least a 1-year history of migraine and onset before 50 years of age. Patients had an episodic or chronic migraine diagnosis as defined by International Classification of Headache Disorders, 3rd edition (beta version) criteria [16]. In EVOLVE-1/EVOLVE-2, patients could take acute treatments, but no migraine preventive treatments were allowed; however, stable doses of topiramate or propranolol were permitted in REGAIN. Patients could not have received onabotulinumtoxinA for a minimum of 4 months prior to inclusion in any of the trials. Key exclusion criteria included history of failure to respond to three or more adequately dosed classes of migraine preventive treatments as defined by the American Academy of Neurology/American Headache Society treatment guidelines level (A) and (B) evidence [17], prior exposure to any CGRP antibody, and having taken a therapeutic antibody in the past year.

\section{Outcome Measures}

The primary outcome for each study was the overall mean change from baseline in the number of monthly migraine headache days during the double-blind treatment periods [13-15]. This report summarizes post hoc analyses of onset of effect, maintenance of effect, and cessation of effect after treatment completion or discontinuation. Detailed statistical analysis methods are described in the original reports [18-20].

For time of onset of effect of galcanezumab, a sequential analysis approach was used. The first step assessed the onset of effect at monthly intervals (i.e., earliest month at which galcanezumab statistically separated from placebo on the basis of mean change from baseline in monthly migraine headache days and maintained that statistical separation at all subsequent months in the treatment period) [18]. If onset occurred at month 1 , then the onset of effect was assessed at weekly intervals within the first month (i.e., earliest week at which galcanezumab statistically separated from placebo on the basis of mean change from baseline in weekly migraine headache days and maintained that statistical separation at all subsequent weeks of month 1 ). If onset occurred at week 1 , then the onset of effect was assessed at daily intervals within the first week by evaluating the proportion of patients with presence of a migraine headache (yes or no) for each of the initial 7 days of treatment. For the weekly and daily onset analysis, galcanezumab treatment groups (120 mg and $240 \mathrm{mg}$ ) were pooled together because both treatment groups received $240 \mathrm{mg}$ as their first monthly loading dose. Furthermore, an exploratory analysis was conducted to evaluate the percentage of patients with new-onset 50\% response at each month of the double-blind treatment period.

Evaluation of maintenance of effect was a comparison of the percentages of galcanezumab- and placebo-treated patients with maintenance of at least a 50\% response for consecutive months at the individual patient level [19]. In the episodic migraine studies, maintenance of response was calculated for individuals with at least three consecutive months including the patient's last month of dosing and for individuals with six consecutive months of sustained response. In the chronic migraine study, maintenance of response was calculated for individuals with three consecutive months. A logistic regression analysis was used for between-treatment group comparisons. The threshold of at least $50 \%$ response was 
selected because this is a common outcome measure used in clinical trials to measure pain reduction and is considered clinically meaningful [21-24].

Cessation of effect was determined during a 4-month post-treatment period following a 6-month double-blind treatment period for patients with episodic migraine [20] and following a 9-month open-label extension period for patients with chronic migraine on the basis of change from baseline in monthly migraine headache days. A 4-month post-treatment period was selected because galcanezumab was last administered 1 month prior to the end of the treatment period, thus allowing five elimination half-lives prior to the end of the study. The half-life of galcanezumab is 27 days (ca. 1 month) [12].

\section{RESULTS}

\section{Patient Baseline Characteristics}

A total of 1773 adult patients with episodic migraine $\quad(n=444$ galcanezumab $120 \mathrm{mg}$; $n=435$ galcanezumab $240 \mathrm{mg} ;$ and $n=894$ placebo for EVOLVE-1 and EVOLVE-2 studies pooled) $[13,14]$ and 1113 patients with chronic migraine $(n=278$ galcanezumab $120 \mathrm{mg}$; $n=277$ galcanezumab $240 \mathrm{mg} ;$ and $n=558$ placebo) were assessed [15]. Baseline demographics and disease characteristics of the episodic and chronic migraine populations show that more than $80 \%$ were female, more than $71 \%$ were Caucasian, had an approximate mean age of 40 years, and had migraine disease duration of 20 years. At baseline, the mean number of monthly migraine headache days was about 9 for patients with episodic migraine and about 19 for patients with chronic migraine. A complete review of patient disposition and baseline characteristics may be found in the secondary publications for the three studies [18-20].

\section{Onset of Effect}

For patients participating in the episodic migraine studies, onset of effect was initially identified at month 1 for the galcanezumab group vs placebo, with significant treatment effects maintained at all subsequent months $(P<0.001)$ (Fig. 1a, b). At month 1 , mean change from baseline in the number of migraine headache days in EVOLVE-1 was - 3.7 and -3.6 for the galcanezumab $120 \mathrm{mg}$ and $240 \mathrm{mg}$ groups, respectively, vs -1.7 for placebo. Corresponding data for EVOLVE-2 was 3.9 and -3.2 for galcanezumab $120 \mathrm{mg}$ and $240 \mathrm{mg}$, respectively, vs -1.2 for placebo [18]. The subsequent analysis by week showed that onset of effect for galcanezumab occurred at week 1, with significant treatment effects maintained at all subsequent weeks in the first month $(P<0.05)$ (Fig. $2 \mathrm{a}, \mathrm{b})$. Across both studies and across each week, the galcanezumab group consistently experienced a mean reduction of -0.9 to -1.1 migraine headache days/ week, which was significantly greater than corresponding reductions of -0.4 to -0.7 for the placebo group. Additional analyses for each of the first 7 days of treatment determined that a greater proportion of patients on galcanezumab did not have a migraine headache the first day after injection and during each following day of week $1(P<0.05)$ (Fig. 3a, b).

For patients with chronic migraine, onset of effect was initially identified as month 1 for the galcanezumab group vs placebo, with significant treatment effects maintained at all subsequent months $(P<0.01)$ (Fig. 1c). At month 1 , mean change from baseline in the number of migraine headache days was -3.8 and -4.0 for the galcanezumab $120 \mathrm{mg}$ and $240 \mathrm{mg}$ groups, respectively, vs -1.6 for placebo. The subsequent analysis by week showed that onset of effect for galcanezumab occurred at week 1 and was maintained at all subsequent weeks in the first month $(P<0.001)$ (Fig. $2 \mathrm{c})$. Across each week, the galcanezumab group consistently experienced a mean reduction of -1.3 to -1.4 migraine headache days/week, which was significantly greater than corresponding reductions of -0.6 to -0.9 for the placebo group. Further analyses during the first week showed that a greater proportion of patients on galcanezumab did not have a migraine headache the first day after injection and during each following day of week $1(P<0.05)$ (Fig. 3c). 
a
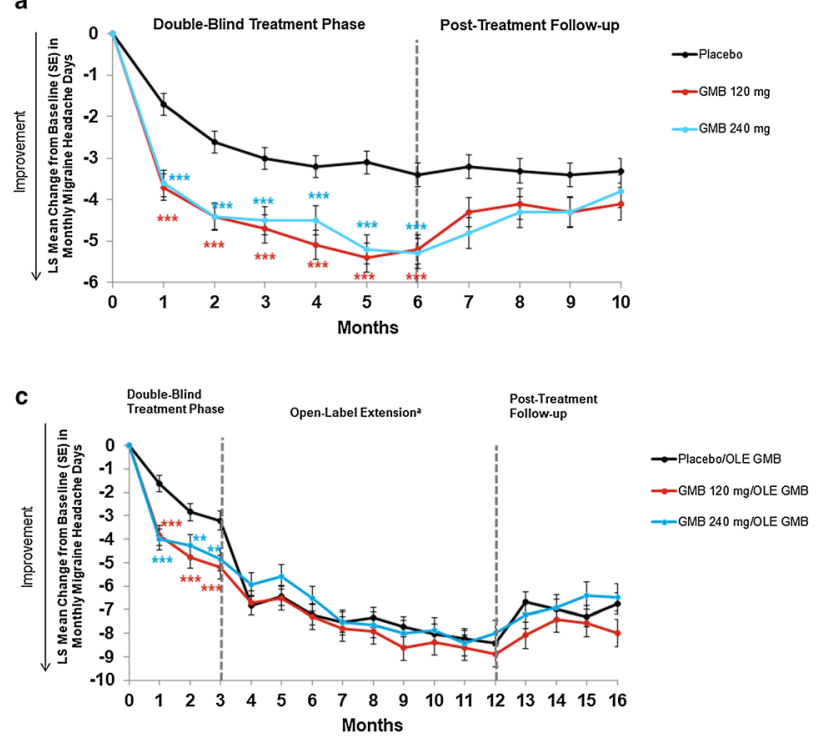

Fig. 1 Onset and cessation of effect by month: a LS mean change from baseline in monthly migraine headache days in the treatment and post-treatment periods of EVOLVE1, b EVOLVE-2, and c REGAIN. ${ }^{* *} P<0.01$; ${ }^{* * *} P<0.001$ vs placebo. ${ }^{\text {a }}$ Patients who entered the openlabel extension period of REGAIN received a $240 \mathrm{mg}$ loading dose of GMB (at month 3), followed by a

a
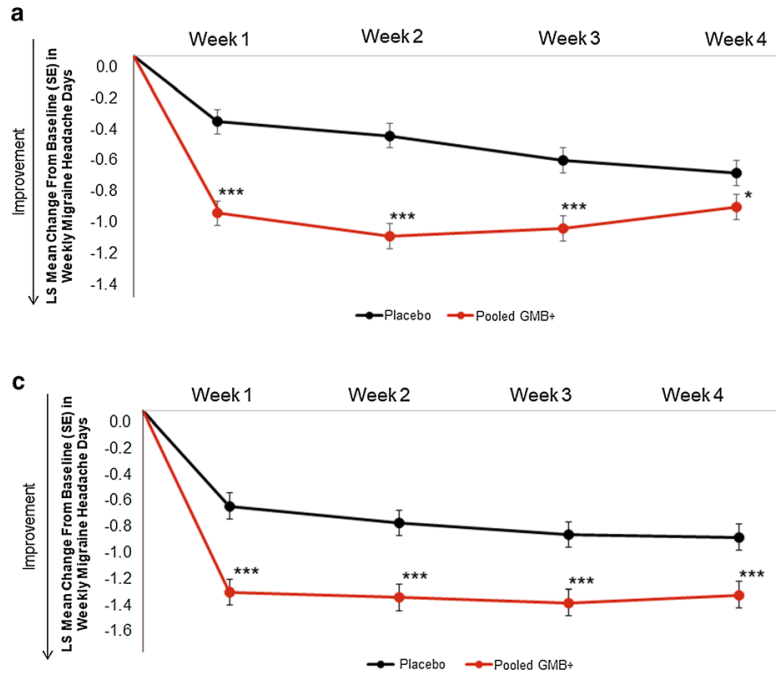

Fig. 2 Onset of effect by week: a LS mean change from 7 -day baseline in weekly migraine headache days for weeks 1-4 of month 1 for EVOLVE-1, b EVOLVE-2, and c REGAIN. ${ }^{*} P<0.05 ;{ }^{* * *} P<0.001$ vs placebo. ${ }^{\phi}$ Onset b

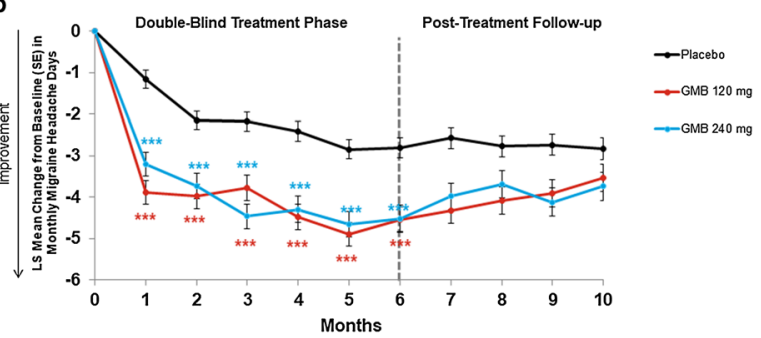

maintenance dose of $120 \mathrm{mg} / \mathrm{month}$ at the next month (month 4), with flexible dosing thereafter throughout the open-label extension (120 or $240 \mathrm{mg} / \mathrm{month}$ ). Treatment assignment is based on the double-blind treatment phase. GMB galcanezumab, LS least squares, OLE open-label extension, SE standard error. Reprinted with permission from [20]

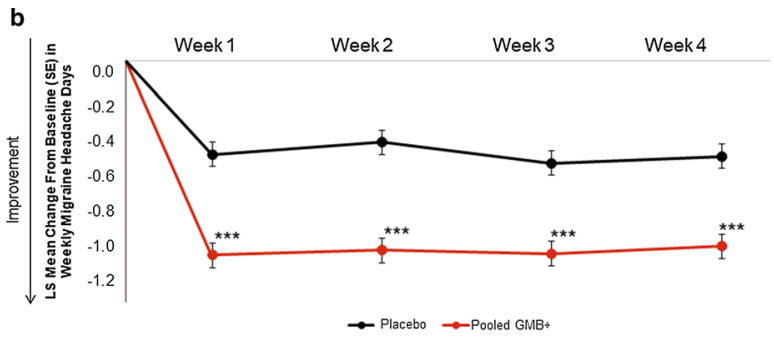

of effect analyses evaluated pooled GMB-treated patients vs placebo (as both GMB groups received $240 \mathrm{mg}$ in the first month). GMB galcanezumab, LS least squares, SE standard error 

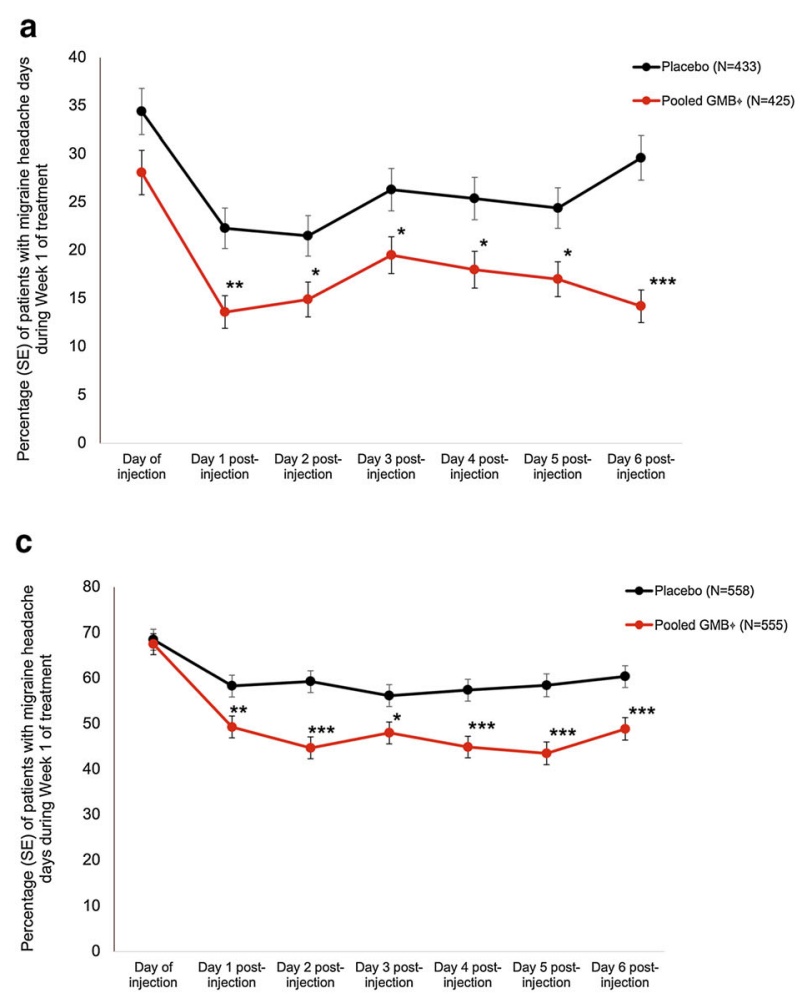

Fig. 3 Onset of effect by day: a Daily estimated proportions of patients with migraine headache days in week 1 of month 1 for EVOLVE-1, b EVOLVE-2, and c REGAIN. ${ }^{*} P<0.05 ;{ }^{* *} P<0.01 ;{ }^{* * *} P<0.001$ vs

A numerically greater proportion of patients in the galcanezumab group achieved new-onset $50 \%$ response compared to placebo. At month 1 , approximately twice as many patients on galcanezumab reached $50 \%$ response vs placebo, regardless of whether they had episodic or chronic migraine. In patients with episodic migraine, $74-79 \%$ of galcanezumabtreated patients had new-onset $50 \%$ response by month 3 compared to $47-55 \%$ on placebo. By month $6,84-88 \%$ of patients with episodic migraine had reached new-onset 50\% response in the galcanezumab group compared to $65-67 \%$ on placebo. In patients with chronic migraine, $44-47 \%$ of galcanezumab-treated patients had new-onset $50 \%$ response by month 3 compared to $29 \%$ on placebo (Table 1 ). b

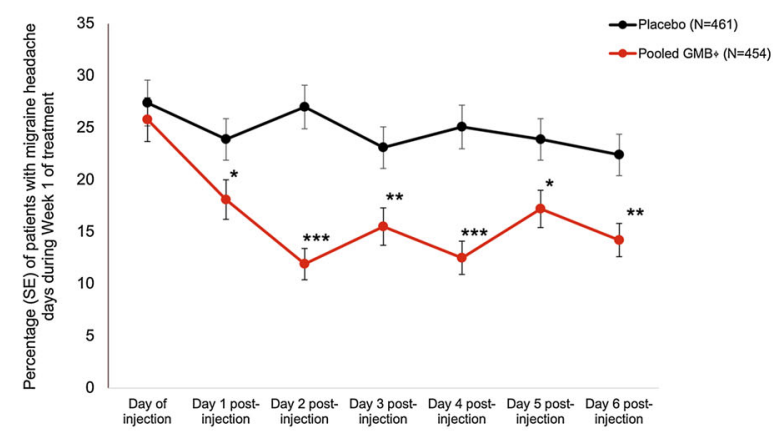

placebo. ${ }^{\phi}$ Onset of effect analyses evaluated pooled GMB-treated patients vs placebo (as both GMB groups received $240 \mathrm{mg}$ in the first month). GMB galcanezumab, $N$ number of intent-to-treat patients, SE standard error

\section{Maintenance of Effect}

For patients with episodic migraine, at least a $50 \%$ response was maintained in $41.5 \%$ and $41.1 \%$ of galcanezumab-treated patients (120 $\mathrm{mg}$ and $240 \mathrm{mg}$, respectively) for at least three consecutive months including the patient's last month of dosing and was significantly greater than the $21.4 \%$ of placebo-treated patients with a similar sustained response $(P<0.001)$ (Fig. 4a) [19]. At least a 50\% response was maintained in $19.0 \%$ and $20.8 \%$ of galcanezumab-treated patients (120 mg and $240 \mathrm{mg}$, respectively) for six consecutive months and was significantly greater than the $8.0 \%$ of placebo-treated patients $(P<0.001)$ (Fig. 4b) [19].

For patients with chronic migraine, at least a $50 \%$ response was maintained in $16.9 \%$ and $14.6 \%$ of galcanezumab-treated patients 
Table 1 Percentage of patients with new-onset 50\% response at each month of the double-blind treatment period for EVOLVE-1, EVOLVE-2, and REGAIN

\begin{tabular}{|c|c|c|c|c|c|c|}
\hline & Month 1 & Month 2 & Month 3 & Month 4 & Month 5 & Month 6 \\
\hline \multicolumn{7}{|l|}{ EVOLVE-1 } \\
\hline \multirow[t]{2}{*}{ Placebo $(N=425)$} & $24.9 \%$ & $17.9 \%$ & $12.2 \%$ & $7.5 \%$ & $2.8 \%$ & $1.4 \%$ \\
\hline & $(24.9 \%)$ & $(42.8 \%)$ & $(55.0 \%)$ & $(62.5 \%)$ & $(65.3 \%)$ & $(66.7 \%)$ \\
\hline \multirow[t]{2}{*}{ GMB $120 \mathrm{mg}(N=210)$} & $49.0 \%$ & $21.0 \%$ & $9.0 \%$ & $5.2 \%$ & $1.4 \%$ & $1.9 \%$ \\
\hline & $(49.0 \%)$ & $(70.0 \%)$ & $(79.0 \%)$ & $(84.2 \%)$ & $(85.6 \%)$ & $(87.5 \%)$ \\
\hline \multirow[t]{2}{*}{ GMB $240 \mathrm{mg}(N=208)$} & $49.0 \%$ & $19.2 \%$ & $6.3 \%$ & $4.8 \%$ & $3.8 \%$ & $0.5 \%$ \\
\hline & $(49.0 \%)$ & $(68.2 \%)$ & $(74.5 \%)$ & $(79.3 \%)$ & $(83.1 \%)$ & $(83.6 \%)$ \\
\hline \multicolumn{7}{|l|}{ EVOLVE-2 } \\
\hline \multirow[t]{2}{*}{ Placebo $(N=450)$} & $22.4 \%$ & $17.3 \%$ & $7.3 \%$ & $8.7 \%$ & $5.3 \%$ & $4.0 \%$ \\
\hline & $(22.4 \%)$ & $(39.7 \%)$ & $(47.0 \%)$ & $(55.7 \%)$ & $(61.0 \%)$ & $(65.0 \%)$ \\
\hline \multirow[t]{2}{*}{ GMB $120 \mathrm{mg}(N=226)$} & $52.2 \%$ & $14.6 \%$ & $7.1 \%$ & $8.4 \%$ & $2.7 \%$ & $1.8 \%$ \\
\hline & $(52.2 \%)$ & $(66.8 \%)$ & $(73.9 \%)$ & $(82.3 \%)$ & $(85.0 \%)$ & $(86.8 \%)$ \\
\hline \multirow[t]{2}{*}{ GMB $240 \mathrm{mg}(N=220)$} & $45.0 \%$ & $16.4 \%$ & $12.3 \%$ & $5.5 \%$ & $4.5 \%$ & $0.0 \%$ \\
\hline & $(45.0 \%)$ & $(61.4 \%)$ & $(73.7 \%)$ & $(79.2 \%)$ & $(83.7 \%)$ & $(83.7 \%)$ \\
\hline \multicolumn{7}{|l|}{ REGAIN } \\
\hline \multirow[t]{2}{*}{ Placebo $(N=538)$} & $11.0 \%$ & $9.7 \%$ & $8.4 \%$ & $\mathrm{~N} / \mathrm{A}$ & N/A & N/A \\
\hline & $(11.0 \%)$ & $(20.7 \%)$ & $(29.1 \%)$ & & & \\
\hline \multirow[t]{2}{*}{ GMB $120 \mathrm{mg}(N=273)$} & $26.4 \%$ & $9.5 \%$ & $7.7 \%$ & $\mathrm{~N} / \mathrm{A}$ & N/A & N/A \\
\hline & $(26.4 \%)$ & $(35.9 \%)$ & $(43.6 \%)$ & & & \\
\hline \multirow[t]{2}{*}{ GMB $240 \mathrm{mg}(N=274)$} & $23.4 \%$ & $12.4 \%$ & $11.3 \%$ & $\mathrm{~N} / \mathrm{A}$ & N/A & $\mathrm{N} / \mathrm{A}$ \\
\hline & $(23.4 \%)$ & $(35.8 \%)$ & $(47.1 \%)$ & & & \\
\hline
\end{tabular}

The numbers in parentheses represent the cumulative percentage of patients with new-onset $50 \%$ response at each month $G M B$ galcanezumab, $N$ number of intent-to-treat patients, $N / A$ not applicable

(120 $\mathrm{mg}$ and $240 \mathrm{mg}$, respectively) and was greater than the $6.3 \%$ of placebo-treated patients $(P<0.001)$ (Fig. 4c) [19]. The difference between dose groups for either episodic or chronic migraine in maintenance of response was not significant.

\section{Cessation of Therapy}

Overall, 740 patients in EVOLVE-1 and 830 patients in EVOLVE-2 entered the post-treatment period. In both studies, month 6 represents the time at which the last dose of galcanezumab was administered. For EVOLVE1 , the average increase in the number of monthly migraine headache days during the post-treatment period was 1.0 day for the $120 \mathrm{mg}$ dose, 1.0 day for the $240 \mathrm{mg}$ dose, and 0.1 days for placebo. For EVOLVE-2, the average increase in the number of monthly migraine headache days during the post-treatment period was 0.6 days for the $120 \mathrm{mg}$ dose, 0.6 days for the $240 \mathrm{mg}$ dose, and 0.1 days for placebo (Fig. 1a, b) [20]. 

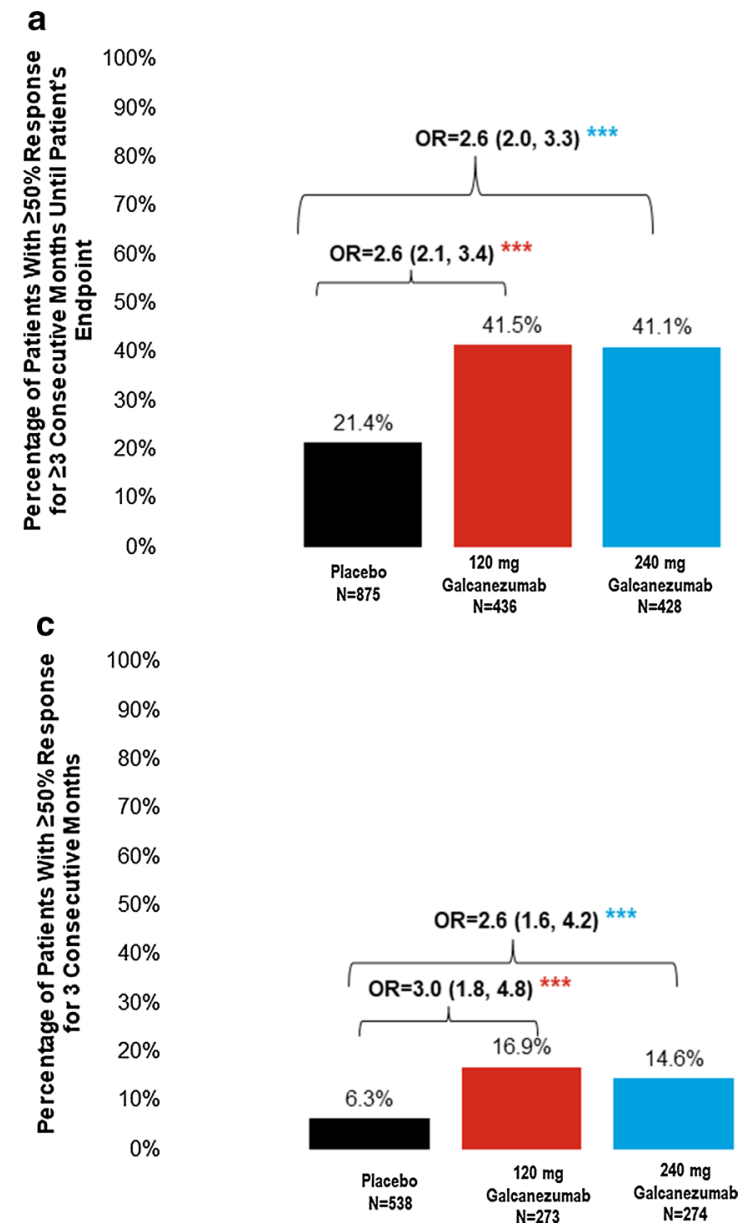

Fig. 4 Maintenance of effect: a Percentages of patients with episodic migraine with maintenance of $\geq 50 \%$ response for $\geq 3$ consecutive months including the patient's last month of dosing. b Percentages of patients with episodic migraine with maintenance of $\geq 50 \%$

For REGAIN, 912 patients entered the posttreatment period. Following treatment cessation, from the end of the open-label treatment period at month 13 to the end of the posttreatment period at month 16, there was a gradual loss of improvement in monthly migraine headache days. Specifically, the average increase in the number of monthly migraine headache days during the post-treatment period was 1.1 days for the $120 \mathrm{mg}$ dose, 1.2 days for the $240 \mathrm{mg}$ dose, and 1.5 days for placebo (Fig. 1c). Given that the open-label extension preceded the post-treatment period, all patients received galcanezumab prior to
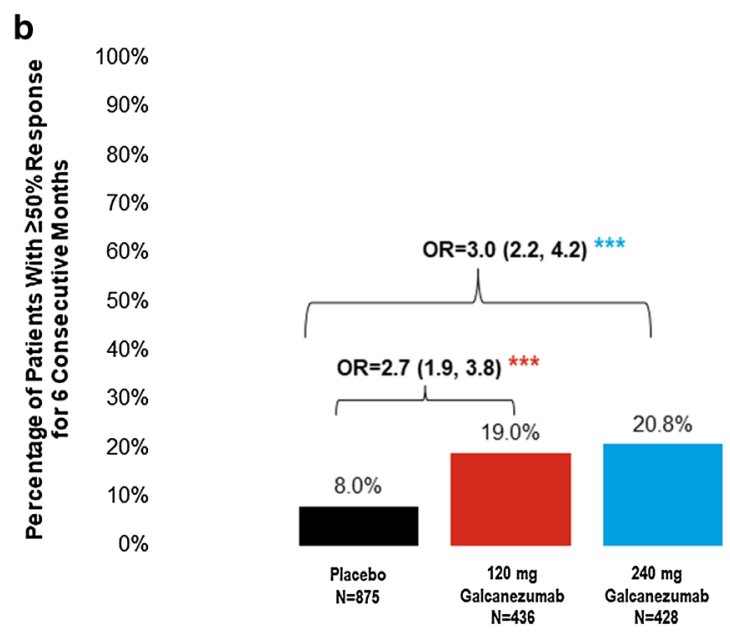

response for 6 consecutive months. c Percentages of patients with chronic migraine with maintenance of $\geq 50 \%$ response for 3 consecutive months. ${ }^{* * *} P<0.001$ vs placebo. OR odds ratio. Reprinted with permission from [19]

treatment cessation and thus all three treatment groups had a similar decline in monthly migraine headache day reduction from month 13 to month 16 .

For all studies, the difference in monthly migraine headache days relative to baseline remained statistically significant throughout the post-treatment period $(P<0.001)$. In EVOLVE-1 and EVOLVE-2, the differences between galcanezumab and placebo were statistically significant throughout the post-treatment period except for month 10 . There were no new adverse events during the post-treatment period. There was no evidence of 
withdrawal or rebound headaches following galcanezumab discontinuation [20].

\section{DISCUSSION}

The present analyses indicate that galcanezumab, a CGRP monoclonal antibody that is used for the preventive treatment of migraine, had an onset of effect beginning as early as the first day after injection for some patients with episodic or chronic migraine; maintenance of effect throughout the treatment period (6 months in episodic migraine and 3 months in chronic migraine); and gradual reduction of effect without signs of rebound headache throughout the 4-month post-treatment period in the majority of patients with episodic and chronic migraine. While results of the $120 \mathrm{mg} / \mathrm{month}$ (with a $240 \mathrm{mg}$ loading dose) and $240 \mathrm{mg} / \mathrm{month}$ treatment arms are reported, the approved dose for migraine prevention is $120 \mathrm{mg}$ monthly following a $240 \mathrm{mg}$ loading dose. In all three clinical trials, the most common adverse reactions were injection site reactions [12].

The time course of effect of galcanezumab has relevance to clinicians and patients. Patient persistence on oral migraine preventive medications is often low [6, 7]. Speed of onset influences patient preference and may improve persistence [25]. Galcanezumab showed an early onset of effect, which was replicated in three large phase 3 clinical trials in adult patients with episodic and chronic migraine. Both doses of galcanezumab (120 mg and $240 \mathrm{mg}$ ) achieved a statistically significant reduction in the number of monthly migraine headache days beginning at month 1 . Additional weekly analyses within the first month showed separation from placebo at week 1 . Daily analyses within the first week found that the estimated proportion of patients experiencing migraine headaches was significantly lower in the galcanezumab group beginning the first day after injection. Further, a numerically greater proportion of patients in the galcanezumab group achieved new-onset $50 \%$ response during the initial months of treatment compared to placebo. However, it is important to note that this early onset of effect is not necessary to achieve longterm efficacy as most guidelines recommend evaluating CGRP monoclonal antibodies for at least 3 months to determine if a patient is responsive $[8,26]$.

Galcanezumab also demonstrated good maintenance of effect. Galcanezumab-treated patients were two times more likely than placebo-treated patients to achieve a sustained response of at least $50 \%$ for three consecutive months in both episodic and chronic migraine studies. There is some evidence that improvement with preventive treatment at 3 months may predict lasting remission [27]. This maintenance of effect also has the potential to improve persistence.

Following cessation of galcanezumab, response rates declined over time but did not return to baseline for patients with episodic and chronic migraine [20]. This persistence of effect is important for patients should they need to temporarily stop preventive treatment or switch medications. No new adverse events, including rebound headache, were observed during the post-treatment period [20].

These findings of early onset, persistence of effect, and gradual decline in efficacy following cessation may be related to the pharmacokinetics of galcanezumab [12]. Studies of the pharmacokinetic/pharmacodynamic properties of galcanezumab have found an average time to peak serum concentration of galcanezumab of 5 days and attainment of therapeutic steadystate concentrations following the loading dose $[12,28]$. The average half-life of 27 days allows for monthly administration of galcanezumab and may play a role in the persistence of effect even after discontinuation [12]. This represents an advantage over some oral therapies, which require daily dosing and long titration schedules to reach a therapeutic dose. Patients may thus discontinue oral treatments before realizing their full potential.

These results were not without limitations. The study population was predominantly middle-aged, Caucasian women. Patients with unstable medical or psychiatric conditions, high body mass index ( $40 \mathrm{~kg} / \mathrm{m}^{2}$ or more), high risk of serious cardiovascular events, and those who had failed three or more adequately dosed 
classes of migraine preventive treatments were excluded. Although these factors may limit generalizability, most patients with episodic and chronic migraine fall within the study spectrum. Several of these analyses are post hoc in nature and the parent studies were not specifically powered for these assessments. However, the results were replicated across three studies.

\section{CONCLUSIONS}

There has been a longstanding need for new effective preventive treatment options for patients with episodic and chronic migraine. Galcanezumab helps fill that void by offering patients with this debilitating disease a migraine-specific therapeutic option that has early onset of action, sustained reduction in migraine headache days over time, and gradual loss of effect upon cessation without evidence of rebound of headache frequency.

\section{ACKNOWLEDGEMENTS}

The authors thank the participants of the studies included in this review.

Funding. Eli Lilly and Company was involved in the following roles: study design; collection, analysis, and interpretation of data; writing of the manuscript; and the decision to submit the article for publication. Eli Lilly and Company is also funding the journal's Rapid Service and Open Access Fees.

Authorship. All named authors below meet the International Committee of Medical Journal Editors (ICMJE) criteria for authorship for this article, take responsibility for the integrity of the work as a whole, and have given their approval for this version to be published.

Authorship Contributions. Category 1: (a) Conception/design: EMP, (b) Acquisition of data: YD, (c) Analysis of data: YD, (d) Interpretation of data: DKK, JMN, AJK, YD, EMP, SLH. Category 2: (a) Drafting article: DKK, AJK, (b) Revising it critically for important intellectual content: DKK, JMN, AJK, YD, EMP, SLH. Category 3: (a) Final approval of the version to be published: DKK, JMN, AJK, YD, EMP, SLH.

\section{Medical Writing, Editorial and Other} Assistance. Dustin D. Ruff, PhD (Eli Lilly and Company), provided statistical analysis and data interpretation support. Writing support was provided by Teresa Tartaglione, PharmD (Synchrogenix, a Certara Company, Wilmington, DE). This medical writing assistance was funded by Eli Lilly and Company.

Disclosures. Dr. Kuruppu, A. Kovacik, PA-C, Dr. Dong, and Dr. Pearlman are employees and minor stockholders of Eli Lilly and Company. Dr. North serves as a consultant for Eli Lilly and Company and is on the speakers bureau for Eli Lilly and Company, Takeda/Lundbeck, and AbbVie. Dr. Hutchinson has served as a consultant for AbbVie, Amgen, Biohaven, Currax, electroCore, Eli Lilly and Company, Lundbeck, Novartis, Teva, Theranica, and Upsher-Smith Pharmaceuticals. She is on the speakers bureau for AbbVie, Amgen, Biohaven, electroCore, Eli Lilly and Company, Novartis, and Teva.

Compliance with Ethics Guidelines. Study protocols were reviewed and approved by the institutional review board for each study site. Studies were conducted according to Good Clinical Practice and to the Helsinki Declaration of 1964 and its later amendments. As this was a retrospective analysis using data that had been previously collected, participants were not required to provide formal Consent to Release Information forms. Consent and ethics reviews were performed in the original studies. A detailed list of ethical review boards can be found in the supplementary material.

Data Availability. Lilly provides access to all individual participant data collected during the trial, after anonymization, with the exception of pharmacokinetic or genetic data. Data are available to request 6 months after the indication studied has been approved in the USA and EU and after primary publication acceptance, whichever is later. No expiration 
date of data requests is currently set once data are made available. Access is provided after a proposal has been approved by an independent review committee identified for this purpose and after receipt of a signed data sharing agreement. Data and documents, including the study protocol, statistical analysis plan, clinical study report, and blank or annotated case report forms, will be provided in a secure data sharing environment. For details on submitting a request, see the instructions provided at http:// www.vivli.org.

Open Access. This article is licensed under a Creative Commons Attribution-NonCommercial 4.0 International License, which permits any non-commercial use, sharing, adaptation, distribution and reproduction in any medium or format, as long as you give appropriate credit to the original author(s) and the source, provide a link to the Creative Commons licence, and indicate if changes were made. The images or other third party material in this article are included in the article's Creative Commons licence, unless indicated otherwise in a credit line to the material. If material is not included in the article's Creative Commons licence and your intended use is not permitted by statutory regulation or exceeds the permitted use, you will need to obtain permission directly from the copyright holder. To view a copy of this licence, visit http://creativecommons.org/licenses/by$\mathrm{nc} / 4.0 /$.

\section{REFERENCES}

1. Lipton RB, Bigal ME, Diamond M, et al. Migraine prevalence, disease burden, and the need for preventive therapy. Neurology. 2007;68:343-9.

2. Headache Classification Committee of the International Headache Society (IHS). The International Classification of Headache Disorders, 3rd edition. Cephalalgia. 2018;38:1-211.

3. GBD 2016 Disease and Injury Incidence and Prevalence Collaborators. Global, regional, and national incidence, prevalence, and years lived with disability for 328 diseases and injuries for 195 countries, 1990-2016: a systematic analysis for the
Global Burden of Disease Study 2016. Lancet. 2017;390:1211-59.

4. Becker WJ. Acute migraine treatment in adults. Headache. 2015;55:778-93.

5. Lipton RB, Silberstein SD. Episodic and chronic migraine headache: breaking down barriers to optimal treatment and prevention. Headache. 2015;55(Suppl 2):103-22.

6. Hepp Z, Dodick DW, Varon SF, et al. Persistence and switching patterns of oral migraine prophylactic medications among patients with chronic migraine: a retrospective claims analysis. Cephalalgia. 2017;37:470-85.

7. Ford JH, Schroeder K, Nyhuis AW, Foster SA, Aurora SK. Cycling through migraine preventive treatments: implications for all-cause total direct costs and disease-specific costs. J Manag Care Spec Pharm. 2019;25:45-59.

8. American Headache Society. The American Headache Society position statement on integrating new migraine treatments into clinical practice. Headache. 2019;59:1-18.

9. Malhotra R. Understanding migraine: potential role of neurogenic inflammation. Ann Indian Acad Neurol. 2016;19:175-82.

10. Raffaelli B, Reuter U. The biology of monoclonal antibodies: focus on calcitonin gene-related peptide for prophylactic migraine therapy. Neurotherapeutics. 2018;15:324-35.

11. Paemeleire K, MaassenVanDenBrink A. Calcitoningene-related peptide pathway mAbs and migraine prevention. Curr Opin Neurol. 2018;31:274-80.

12. Emgality $^{\mathrm{TM}}$ (galcanezumab-gnlm) [package insert]. Indianapolis: Eli Lilly and Company; 2019.

13. Stauffer VL, Dodick DW, Zhang Q, Carter JN, Ailani J, Conley RR. Evaluation of galcanezumab for the prevention of episodic migraine: the EVOLVE-1 randomized clinical trial. JAMA Neurol. 2018;75: 1080-8.

14. Skljarevski V, Matharu M, Millen BA, Ossipov MH, Kim BK, Yang JY. Efficacy and safety of galcanezumab for the prevention of episodic migraine: results of the EVOLVE-2 phase 3 randomized controlled clinical trial. Cephalalgia. 2018;38:1442-54.

15. Detke HC, Goadsby PJ, Wang S, Friedman DI, Selzler KJ, Aurora SK. Galcanezumab in chronic migraine: the randomized, double-blind, placebocontrolled REGAIN study. Neurology. 2018;91: e2211-21. 
16. Headache Classification Committee of the International Headache Society (IHS). The International Classification of Headache Disorders, 3rd edition (beta version). Cephalalgia. 2013;33:629-808.

17. Silberstein SD, Holland S, Freitag F, Dodick DW, Argoff C, Ashman E. Evidence-based guideline update: pharmacologic treatment for episodic migraine prevention in adults: report of the Quality Standards Subcommittee of the American Academy of Neurology and the American Headache Society. Neurology. 2012;78:1337-45.

18. Detke HC, Millen BA, Zhang Q, et al. Rapid onset of effect of galcanezumab for the prevention of episodic migraine: analysis of the EVOLVE studies. Headache. 2020;60:348-59.

19. Förderreuther S, Zhang Q, Stauffer VL, Aurora SK, Láinez MJA. Preventive effects of galcanezumab in adult patients with episodic or chronic migraine are persistent: data from the phase 3, randomized, double-blind, placebo-controlled EVOLVE-1, EVOLVE-2, and REGAIN studies. J Headache Pain. 2018;19:121.

20. Stauffer VL, Wang S, Voulgaropoulos M, Skljarevski V, Kovacik A, Aurora SK. Effect of galcanezumab following treatment cessation in patients with migraine: results from 2 randomized phase 3 trials. Headache. 2019;59:834-47.

21. Tfelt-Hansen P, Pascual J, Ramadan N, et al. Guidelines for controlled trials of drugs in migraine: third edition. A guide for investigators. Cephalalgia. 2012;32:6-38.
22. Diener HC, Bussone G, Van Oene JC, et al. Topiramate reduces headache days in chronic migraine: a randomized, double-blind, placebo-controlled study. Cephalalgia. 2007;27:814-23.

23. Dodick DW, Ashina M, Brandes JL, et al. ARISE: a phase 3 randomized trial of erenumab for episodic migraine. Cephalalgia. 2018;38:1026-37.

24. Silberstein SD, Dodick DW, Aurora SK, et al. Percent of patients with chronic migraine who responded per onabotulinumtoxinA treatment cycle: PREEMPT. J Neurol Neurosurg Psychiatry. 2015;86: 996-1001.

25. Peres MFP, Silbertstein S, Moreira F, et al. Patients' preference for migraine preventive therapy. Headache. 2007;47:540-5.

26. Sacco S, Bendtsen L, Ashina M, et al. European headache federation guideline on the use of monoclonal antibodies acting on the calcitonin gene related peptide or its receptor for migraine prevention. J Headache Pain. 2019;20:6.

27. Bhoi SK, Kalita J, Misra UK. Is 6 months of migraine prophylaxis adequate? Neurol Res. 2013;35: 1009-14.

28. Kielbasa W, Helton D. A new era for migraine: pharmacokinetic and pharmacodynamic insights into monoclonal antibodies with a focus on galcanezumab, an anti-CGRP antibody. Cephalalgia. 2019;39:1284-97. 